\title{
Increases in Doublecortin Immunoreactivity in the Dentate Gyrus following Extinction of Heroin-Seeking Behavior
}

\author{
Megan P. Hicks, ${ }^{1}$ Kelly C. Wischerath, ${ }^{2}$ Amber L. Lacrosse, ${ }^{2}$ and M. Foster Olive ${ }^{2,3}$ \\ ${ }^{1}$ Department of Psychiatry and Behavioral Sciences, Medical University of South Carolina, Charleston, SC 29425, USA \\ ${ }^{2}$ Department of Psychology, Arizona State University, 950 S. McAllister Avenue, P.O. Box 871104, Tempe, AZ 85287, USA \\ ${ }^{3}$ Interdisciplinary Graduate Program in Neuroscience, Arizona State University, Tempe, AZ 85287, USA
}

Correspondence should be addressed to M. Foster Olive, foster.olive@asu.edu

Received 16 June 2012; Revised 3 October 2012; Accepted 6 October 2012

Academic Editor: Chitra D. Mandyam

Copyright (C) 2012 Megan P. Hicks et al. This is an open access article distributed under the Creative Commons Attribution License, which permits unrestricted use, distribution, and reproduction in any medium, provided the original work is properly cited.

\begin{abstract}
Adult-generated neurons in the dentate gyrus (DG) of the hippocampus play a role in various forms of learning and memory. However, adult born neurons in the DG, while still at an immature stage, exhibit unique electrophysiological properties and are also functionally implicated in learning and memory processes. We investigated the effects of extinction of drug-seeking behavior on the formation of immature neurons in the DG as assessed by quantification of doublecortin (DCX) immunoreactivity. Rats were allowed to self-administer heroin $(0.03 \mathrm{mg} / \mathrm{kg} /$ infusion $)$ for 12 days and then subjected either to 10 days of extinction training or forced abstinence. We also examined extinction responding patterns following heroin self-administration in glial fibrillary acidic protein thymidine kinase (GFAP-tk) transgenic mice, which have been previously demonstrated to show reduced formation of immature and mature neurons in the DG following treatment with ganciclovir (GCV). We found that extinction training increased DCX immunoreactivity in the dorsal DG as compared with animals undergoing forced abstinence, and that GCV-treated GFAP-tk mice displayed impaired extinction learning as compared to saline-treated mice. Our results suggest that extinction of drug-seeking behavior increases the formation of immature neurons in the DG and that these neurons may play a functional role in extinction learning.
\end{abstract}

\section{Introduction}

Adult-born neurons in the dentate gyrus (DG) of the adult hippocampus are generated from neural progenitor cells (NPCs) located in the subgranular layer of this brain region, which proliferate and differentiate first into immature neurons and subsequently migrate into the granule cell layer as mature neurons where they integrate into existing hippocampal circuits [1]. There are numerous lines of evidence to support the notion that mature adult-born neurons contribute to several forms of hippocampus-dependent learning and memory processes such as spatial navigation, contextual learning, and pattern separation (reviewed in [27]). Various neuropsychiatric disorders such as depression, schizophrenia, and drug addiction are characterized by alterations in neurogenesis in the DG $[1,8-10]$. In the drug addiction literature, there is general agreement that most drugs of abuse, including opiates, ethanol, and psychostimulants, suppress neurogenesis in the DG [11-16], and it has been suggested that diminished DG neurogenesis may play a causative role in certain cognitive deficits frequently observed in drug addicts, such as maladaptive contextual and episodic memory formation, altered spatial abilities, cognitive inflexibility, and susceptibility to relapse [17-19].

While the majority of research on neurogenesis in the adult DG has focused on factors influencing the proliferation, differentiation, and survival of NPCs, as well as the role of mature adult-born neurons in various cognitive functions, relatively little is known about the function of adult-born neurons that have not yet fully developed into mature granule cells. There are known species-specific time periods (approximately 3-6 weeks in rodents) between NPC proliferation and formation of mature dentate granule cells when adult-born neurons are considered immature. During 
this time period, immature adult-born neurons undergo a substantial amount of morphological and physiological maturation and exhibit enhanced synaptic plasticity and lower thresholds for the induction of long-term potentiation (LTP) $[20,21]$. During this stage, immature neurons transiently express the microtubule-associated protein doublecortin (DCX) $[22,23]$, which allows for immunohistochemical detection and quantification of the number of immature neurons in the DG. Recent studies using novel transgenic approaches to selectively ablate immature neurons in the DG have revealed that in fact these neurons mediate spatial memory as well as the extinction of conditioned fear [24]. However, the role of immature DG neurons in extinction of addiction-related behaviors has not yet been explored.

Given that extinction is an established form of new and active learning [25-28], the goal of the present study was designed to explore the possibility that immature DG neurons are involved in the extinction of drug-seeking behavior. In Experiment 1, we sought to determine if extinction of drug-seeking behavior following heroin selfadministration would alter DCX immunoreactivity in the DG as compared to animals undergoing forced abstinence. In Experiment 2, we sought to determine if suppression of the generation of immature neurons in the DG would affect extinction responding following heroin self-administration.

\section{Material and Methods}

2.1. Subjects. Animals were maintained on a 12-hour lightdark cycle (lights off at 07:00 hr) in a temperature- and humidity-controlled room. Animals were given ad libitum access to food and water during all phases of the experiment except during behavioral testing. All experimental procedures were conducted with the approval of the Institutional Animal Care and Use Committee of the Medical University of South Carolina and in accordance with the Principles of Laboratory Animal Care and the Guidelines for the Care and Use of Mammals in Neuroscience and Behavioral Research (National Research Council, 2003). For Experiment 1, male Sprague-Dawley rats (Harlan Laboratories, Livermore, CA, USA) weighing approximately $250-275 \mathrm{~g}$ were individually housed upon arrival. For Experiment 2, male GFAP-tk mice (B6.Cg-Tg(Gfap-Tk) 7.1 Mvs/J, stock \#005698; Jackson Laboratories, Bar Harbor, ME, USA) weighing approximately $20-25 \mathrm{~g}$ were individually housed upon arrival. This mouse strain was generated as described elsewhere $[29,30]$ to express a herpes simplex virus thymidine kinase (tk) exclusively in GFAP-positive cells, and proliferating cells expressing the transgene in the presence of the antiviral agent ganciclovir (GCV) produce toxic nucleotide analogues that promote cell death. Thus, GFAP-tk mice have been used as a tool for investigating the function of adult-generated neuronal precursors [31]. This strain has been backcrossed from the founder line onto a C57BL/6 background strain for more than 12 generations. Prior to shipment, all animals were implanted with jugular vein catheters by Harlan or Jackson Laboratories Surgical Services. Catheters were filled with a Hep-Lock solution, tunneled subcutaneously to exit the dorsum between the scapulae, plugged with $1 \mathrm{~cm}$ segments of stainless steel tubing and secured to skin on the dorsum with wound clips.

2.2. Drugs. Heroin (diacetylmorphine hydrochloride), 5bromodeoxyuridine (BrDU), and sodium pentobarbital were obtained from Sigma-Aldrich (St. Louis, MO, USA). Heroin was dissolved in sterile saline for intravenous (i.v.) selfadministration. BrDU was dissolved in sterile saline at a concentration of $50 \mathrm{mg} / \mathrm{mL}$ for intraperitoneal (i.p.) administration and was administered in a volume of $1 \mathrm{~mL} / \mathrm{kg}$ to yield a final dose of $50 \mathrm{mg} / \mathrm{kg}$ per injection. Sodium pentobarbital was dissolved in sterile saline containing $10 \% \mathrm{v} / \mathrm{v}$ ethanol and $40 \% \mathrm{v} / \mathrm{v}$ propylene glycol at a concentration of $150 \mathrm{mg} / \mathrm{mL}$ for i.p. administration. Ganciclovir (GCV) was obtained from Waterstone Technology (St. Carmel, IN, USA) and dissolved at a concentration of $10 \mathrm{mg} / \mathrm{mL}$ in sterile saline for subcutaneous administration via osmotic minipumps.

2.3. Apparatus. Drug self-administration and extinction training were conducted in operant self-administration chambers (ENV-008 for rats, ENV-307 for mice; Med Associates, St. Albans, VT, USA). Each self-administration chamber was located inside a sound-attenuating cubicle equipped with a house light and an exhaust fan designed to mask external noise and odors and was interfaced to a PC computer. Positioned above each lever was a $2.5 \mathrm{~cm}$ diameter white stimulus light. Syringe pumps were located outside self-administration chambers and were interfaced to a PC. Drug solutions were delivered via the syringe pump through a single-channel liquid swivel mounted atop of the chamber, which was connected to a vascular access port.

2.4. Surgical Procedures. Upon arrival, animals were allowed one day of acclimation before surgical procedures. For Experiment 1 , rats were anesthetized with isoflurane $(2 \% \mathrm{v} / \mathrm{v})$ vaporized in medical-grade oxygen at a flow rate of $2 \mathrm{~L} / \mathrm{min}$. Staples surrounding catheter tubing were removed and a one-inch longitudinal incision was made for implantation of a threaded vascular access port (Plastics One; Roanoke, VA, USA). Access ports were attached to a mesh collar that was sutured underneath the surrounding tissue within the incision and were sealed with a piece of Tygon tubing closed at one end and a protective cap. Following surgical procedures, rats were given 5 days of postoperative care during which they received daily intravenous infusions of $70 \mathrm{U} / \mathrm{mL}$ heparin $(0.2 \mathrm{~mL}$ volume $)$ to maintain catheter patency and $100 \mathrm{mg} / \mathrm{mL}$ cefazolin ( $0.1 \mathrm{~mL}$ volume) to protect against infection. Rats also received daily subcutaneous injections of $2.5 \mathrm{mg} / \mathrm{mL}$ of meloxicam $(0.15 \mathrm{~mL}$ volume $)$ for surgery-related discomfort. The surgery site was also treated with topical lidocaine and triple antibiotic ointment to facilitate healing of the wound.

For Experiment 2, mice were anesthetized as described above, the catheter exit wound was enlarged to $1 \mathrm{~cm}$ in length, and mice were implanted with sterile osmotic minipumps (Model 1004, pump rate $0.11 \mu \mathrm{L} / \mathrm{hr}$; Alzet; Cupertino, CA, USA) containing either sterile saline or 

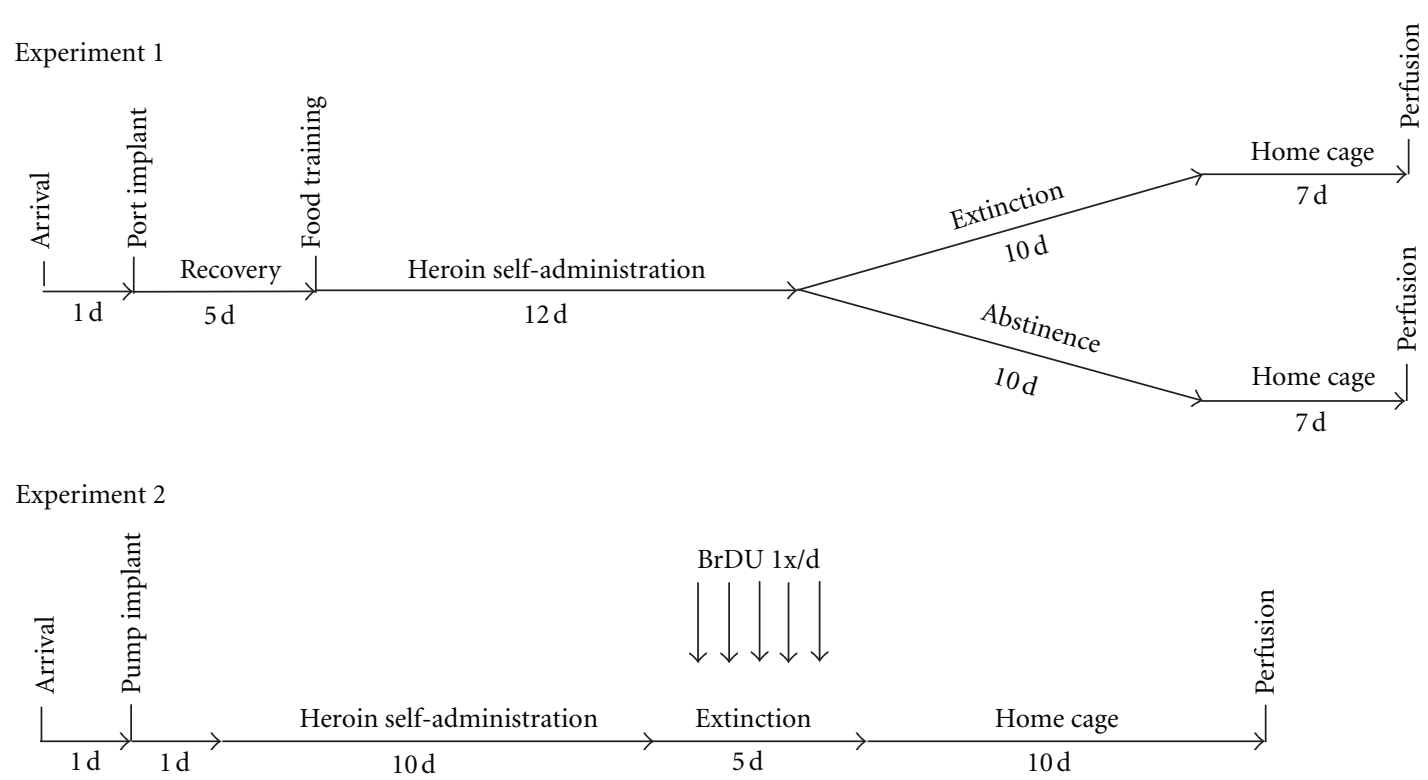

Figure 1: Timelines of procedures in Experiments 1 and 2.

GCV $(10 \mathrm{mg} / \mathrm{mL})$ into the dorsum. The catheter was trimmed to extend $1 \mathrm{~cm}$ from the exit wound. Following minipump implantation, mice were treated with $10 \mathrm{U} / \mathrm{mL}$ heparin (0.05 $\mathrm{mL}$ volume) to maintain catheter patency and $10 \mathrm{mg} / \mathrm{mL}$ cefazolin $(0.05 \mathrm{~mL}$ volume $)$ to protect against infection. The surgery site was treated with topical lidocaine and triple antibiotic ointment to facilitate healing of the wound, and mice were allowed 1 day to recover prior to initiation of drug self-administration procedures.

2.5. Heroin Self-Administration. All self-administration procedures were conducted during the dark phase of the lightdark cycle. A timeline of both experiments is provided in Figure 1. In Experiment 1, to initiate operant responding, rats were placed in the self-administration chambers for a single $16 \mathrm{hr}$ overnight training sessions whereby each press on the designated active lever delivered a $45 \mathrm{mg}$ food pellet (Test-diet, Richmond, IN, USA) into a pellet receptacle on a fixed-ratio 1 (FR1) schedule of reinforcement. Each lever press was accompanied by a concurrent illumination of a stimulus light located above the active lever for $2 \mathrm{sec}$. Each food pellet delivery was followed by a $20 \mathrm{sec}$ time-out period, during which additional active lever presses were recorded but produced no programmed consequences. Presses on the designated inactive lever were recorded but produced no consequences at any time during the experiment. Approximately $24 \mathrm{hr}$ following the initial overnight training session, $3 \mathrm{hr}$ daily heroin self-administration sessions were initiated, whereby presses on the active lever resulted in delivery of heroin $(0.03 \mathrm{mg} / \mathrm{kg} /$ infusion, delivered in a volume of $0.06 \mathrm{~mL}$ over a $2 \mathrm{sec}$ period) on a FR1 schedule of reinforcement. Each drug infusion was followed by a $20 \mathrm{sec}$ timeout period, during which additional active lever presses were recorded but produced no programmed consequences. Each drug infusion was accompanied by concurrent illumination of a stimulus light located above the active lever for $2 \mathrm{sec}$. Self-administration sessions were conducted 6 consecutive days per week for a total of 12 days. Body weights were assessed every 5-7 days in order to assess potential weight loss as a result of heroin self-administration and to adjust the concentration of heroin in the infusion syringe as needed.

In Experiment 2, heroin self-administration in GFAPtk mice was commenced on the day following minipump implantation. Procedures were similar to those of Experiment 1, with the following exceptions: (1) food pellet training was not conducted since mouse self-administration chambers were not equipped with pellet dispensers or receptacles; (2) heroin was delivered in a volume of $0.02 \mathrm{~mL}$ over a $1 \mathrm{sec}$ period; (3) self-administration sessions were conducted for a total of 10 days. Group body weights were assessed on the day following minipump implantation and again during each of the 5 extinction days in order to assess potential weight loss as a result of heroin self-administration and/or GCV treatment and to adjust the concentration of heroin in the infusion syringe as needed. BrDU was administered at a dose of $50 \mathrm{mg} / \mathrm{kg}$ within 1 minute of the end of each of the 5 extinction sessions.

2.6. Extinction Procedures. Extinction sessions were $3 \mathrm{hr}$ in length during which responding on the lever that previously delivered heroin no longer produced any programmed consequences. In addition, the light cue that previously accompanied reinforcer delivery was not available during extinction sessions. Animals were subjected to extinction training to achieve a target level of extinction responding equivalent to $25 \%$ of the number of active lever presses that were emitted on the average of the last 2 days of active heroin self-administration. Since the majority of extinction of responding on the active lever in Experiment 1 was observed during the first 5 extinction training sessions, 
only 5 extinction sessions were conducted in Experiment 2. Immediately following each of the 5 extinction training sessions, mice were injected with $\mathrm{BrDU}$ ( $50 \mathrm{mg} / \mathrm{kg}$ i.p.) to provide an indicator of suppression of the formation of immature neurons.

In Experiment 1, animals undergoing forced abstinence instead of extinction training were transported to a neutral room at approximately the same time of day as when extinction sessions were conducted, handled briefly, and left undisturbed in the home cage for $3 \mathrm{hr}$ (equivalent to the length of extinction sessions). Rats were then returned to the colony room. This process was repeated daily for a total of 10 days to parallel the number of days that extinction training was conducted.

2.7. Assessment of DCX Immunoreactivity. In Experiment 1 , rats were anesthetized with sodium pentobarbital ( $150 \mathrm{mg} / \mathrm{kg}$ i.p.) 7 days following the final extinction training session (or on the same day for animals undergoing forced abstinence-see Figure 1) and perfused transcardially with $100 \mathrm{~mL}$ of phosphate-buffered saline (PBS, $\mathrm{pH}=7.4$ ) containing $0.1 \% \mathrm{w} / \mathrm{v}$ heparin followed by $200 \mathrm{~mL}$ of $4 \% \mathrm{w} / \mathrm{v}$ paraformaldehyde $(\mathrm{pH}=7.4)$ in $0.1 \mathrm{M}$ PBS. Brains were removed, postfixed in the fixative solution for $24 \mathrm{hr}$ at $4^{\circ} \mathrm{C}$, cryoprotected in $30 \% \mathrm{w} / \mathrm{v}$ sucrose in PBS for $48 \mathrm{hr}$ at $4^{\circ} \mathrm{C}$, cut into $30 \mu \mathrm{m}$ coronal sections along the hippocampal neuraxis), and processed for free-floating DCX immunohistochemistry. Sections were preblocked for $1 \mathrm{hr}$ in PBS containing $0.1 \%$ Tween 20, $1 \mathrm{M}$ glycine, and 5\% w/v donkey serum, followed by overnight incubation with rabbit antiDCX primary antisera (1:500; Abcam; Cambridge, MA, USA) at $4^{\circ} \mathrm{C}$. On the next day, sections were washed, incubated with DyLight 594-conjugated donkey anti-rabbit secondary antisera (1:500; Jackson ImmunoResearch; West Grove, PA, USA), washed, mounted onto microscope slides, and coverslipped with Prolong antifade mounting medium (Invitrogen; Carlsbad, CA, USA). Slides were stored in darkness until quantification, which was performed under epifluorescence microscopy at 400x magnification (Leica Microsystems; Bannockburn, IL, USA) by an investigator blind to experimental condition. Manual rotation of the fine focus in the $z$-place was performed to verify the presence of DCX-positive cell bodies. The number of DCX immunoreactive neurons in the subgranular layer of the DG was quantified in one hemisphere from -1.8 to $-6.4 \mathrm{~mm}$ posterior to bregma to the nearest $0.2 \mathrm{~mm}$ according to [32]. Total DCX counts throughout the hippocampal neuraxis were calculated by summation of all counts at each coronal level of section for each individual animal. Images of DCX immunoreactivity in the DG that are presented in Figure 2 were obtained on a Zeiss LSM 410 confocal microscope.

Immunohistochemical staining of tissue from GFAP-tk mice for DCX immunoreactivity yielded inadequate and unsatisfactory staining patterns, as evidenced by a lack of staining of dendritic processes in the granule cell layer. Use of primary antisera from a different vendor also produced unsatisfactory results. We therefore attempted to verify reductions in neuron production in the DG of these mice induced by GCV using previously published procedures for BrDU immunohistochemistry [33] throughout the mouse hippocampal neuraxis.

2.8. Statistical Analyses. Active and inactive lever press data were analyzed by a one-way ANOVA followed by the HolmSidak post hoc tests. DCX immunoreactivity quantified along the hippocampal neuraxis was analyzed by a twoway ANOVA (with the treatment group as the intersubject variable and plane of section as the intrasubject variable) followed by the Holm-Sidak post hoc tests. Total DCX counts in animals undergoing extinction versus abstinence, as well as body weights of mice treated with either saline or GCV, were analyzed by one-way ANOVA. The level of statistical significance was set to $P<0.05$ for all tests.

\section{Results}

The timelines for behavioral procedures in Experiments 1 and 2 are presented in Figure 1. Heroin self-administration data and the effects of extinction versus abstinence on DCX immunoreactivity are depicted in Figure 2. No differences in the number of active or inactive lever presses during active heroin self-administration were observed between animals that subsequently underwent extinction or abstinence $(P>$ 0.05). In addition, the number of heroin infusions obtained in each session did not differ between groups $(P>0.05$, data not shown), indicating that heroin intake was equivalent prior to extinction. Animals subjected to extinction training displayed significant changes in active lever pressing behavior across extinction sessions $(F(10,133)=30.2, P<0.001$; Figure $2(\mathrm{a}))$. Presses on the active lever were significantly increased on the first day of extinction as compared to the average of the last 2 days of active heroin self-administration, characteristic of an expected "extinction burst" following removal of heroin as the reinforcer. After the 2nd day of extinction, lever pressing declined and was significantly lower during the 3rd through 10th extinction sessions as compared to the average of the last two days of selfadministration. The number of inactive lever presses did not differ across experimental groups and also did not change across extinction sessions ( $P$ 's $>0.05$; Figure $2(\mathrm{~b})$ ). The lack of reduction of inactive lever presses produced by extinction training was likely due to a floor effect since the mean number of inactive lever presses per session during the final two days of heroin self-administration was less than 5 for the two groups.

Analysis of DCX immunoreactivity along the hippocampal neuraxis in animals that underwent either extinction training or abstinence following heroin self-administration revealed that extinction-trained animals showed significantly higher levels of DCX immunoreactivity $(F(1,208)=21.95$, $P<0.001$; Figures 2(c)-2(e)) in dorsal regions of the DG ranging from 3.0 to $4.0 \mathrm{~mm}$ posterior to bregma. No group differences were detected in ventral/posterior regions of the DG. In addition, total DCX counts throughout all sections analyzed were higher $(F(1,13)=8.19, P<0.05)$ in animals 


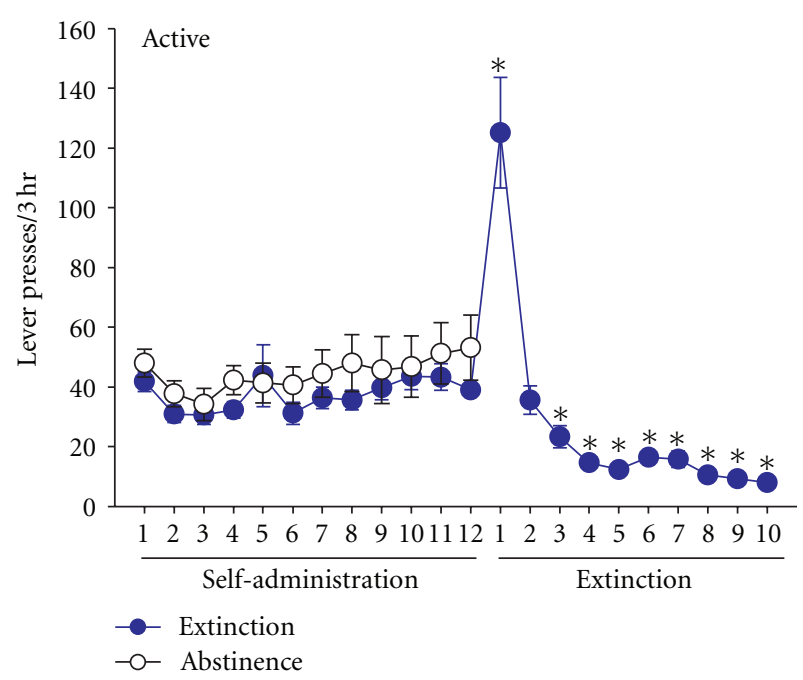

(a)

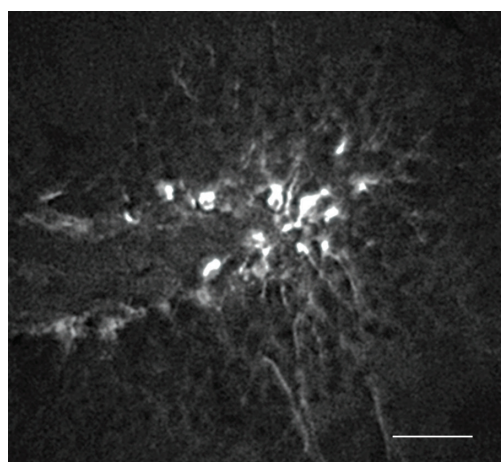

Extinction

(c)

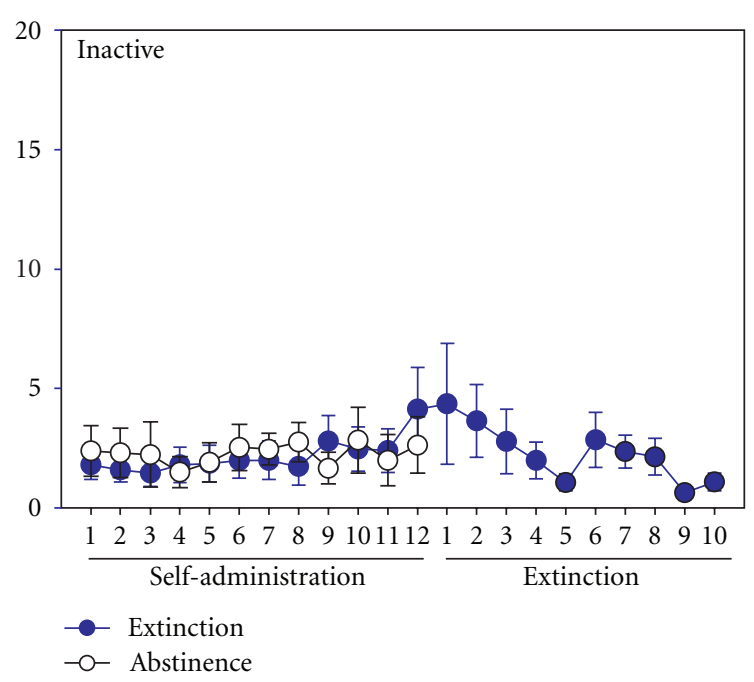

(b)

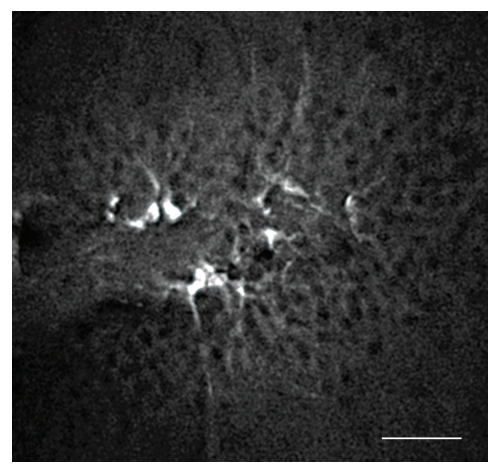

Abstinence

(d)

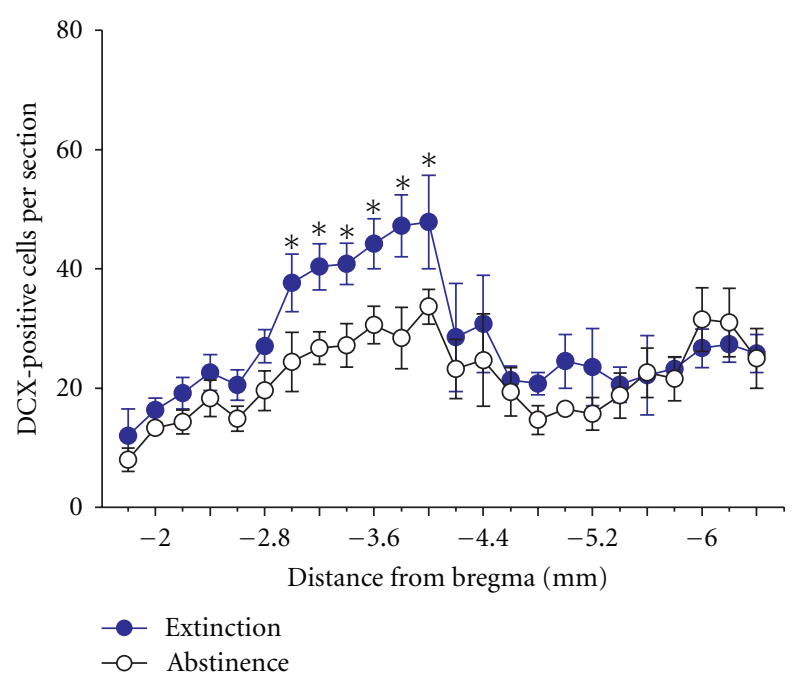

(e)

FIGURE 2: Extinction training increases DCX immunoreactivity in the DG following heroin self-administration (Experiment 1). (a) Active and (b) inactive lever presses during heroin self-administration and extinction. Animals undergoing extinction $(n=15)$ were subjected to 10 days of extinction training following the last day of heroin self-administration, while animals undergoing forced abstinence $(n=13)$ remained in the home cage. ${ }^{*} P<0.05$ versus the average of the last two days of heroin self-administration. (c) and (d) Representative DCX immunostaining at 400x magnification in the DG $(\sim 3.8 \mathrm{~mm}$ posterior to bregma) from animals subjected to extinction (c) or abstinence (d). Scale bar $=35 \mu \mathrm{m}$. (e) Quantification of DCX immunoreactivity along the DG neuraxis in a subset of animals subjected to extinction or abstinence ( $n=8$ per group). ${ }^{*} P<0.05$ versus the abstinence group at the same coronal plane relative to bregma. 


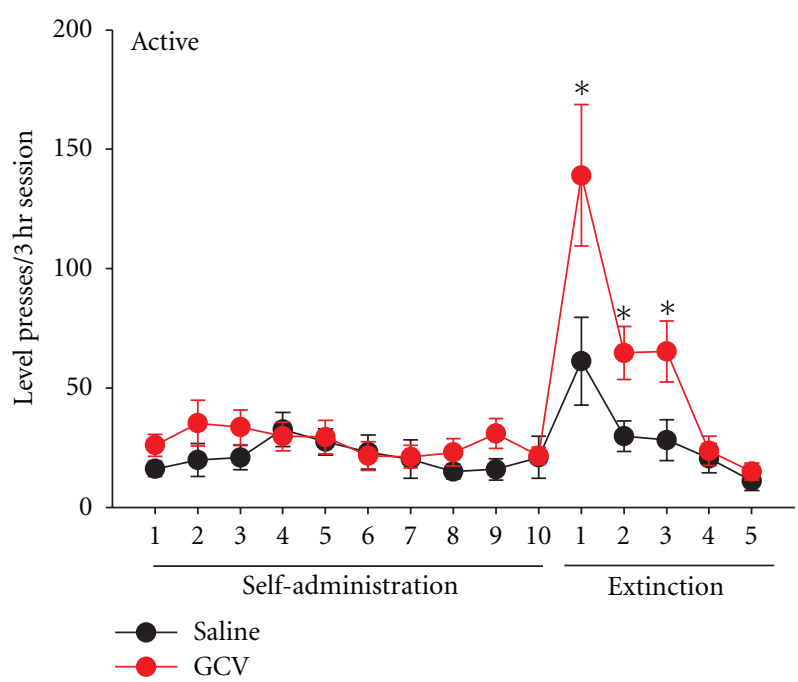

(a)

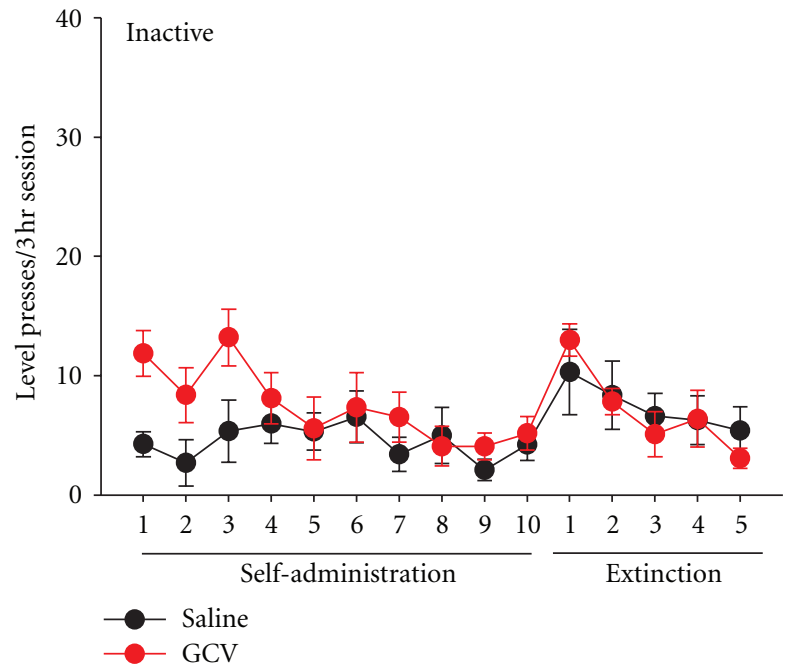

(b)

FIgURE 3: Heroin self-administration and extinction in GFAP-tk mice implanted with minipumps containing either saline or GCV. (a) Active lever presses during 10 days of heroin self-administration followed by 5 days of extinction. Mice treated with GCV ( $n=11)$ displayed increased extinction responding during the first 3 extinction days (E1-E3) as compared to mice that were treated with saline $(n=9)$. ${ }^{*} P<0.05$ versus saline-treated mice on the same day of extinction. (b) Inactive lever presses during 10 days of heroin self-administration followed by 5 days of extinction.

that underwent extinction $(502.4 \pm 45.6$, mean \pm SEM $)$ than those that underwent abstinence $(357.2 \pm 25.8$, mean \pm SEM).

The results of Experiment 2 are depicted in Figure 3. No differences in lever pressing during the 10 days of active heroin self-administration were noted in GFAP-tk mice receiving saline or GCV $(P>0.05$; Figure $3(\mathrm{a}))$ or in the number of heroin infusions obtained $(P>0.05$, data not shown) indicating equal levels of heroin intake between the two groups. Levels of heroin intake in these GFAP-tk mice were similar to those reported previously in $\mathrm{Balb} / \mathrm{c}$ mice [34]. On the first 3 days of extinction, GCVtreated mice displayed an increased number of presses on the lever that previously delivered heroin $(F(1,226)=$ 5.8, $P<0.05$; Figure 3(a)), suggesting an impairment in extinction learning. No differences between treatment groups were noted for inactive lever presses across any phase of the experiment $(P>0.05$; Figure $3(\mathrm{~b}))$. Similarly, analysis of body weight data before and after heroin selfadministration revealed neither weight loss nor weight gain $(P>0.05)$, suggesting that heroin self-administration and/or GCV treatment did not produce nonspecific effects on food intake in GFAP-tk mice.

As mentioned earlier, use of primary antisera against DCX from two different commercial vendors produced inadequate and unsatisfactory staining patterns. Therefore, to verify the GCV-induced reduction of newly proliferating cells in the DG of GFAP-tk mice, immunohistochemistry for BrDU was performed. The number of BrDU-positive cells per section was significantly lower in mice treated with GCV $(5.7 \pm 0.6$, mean \pm SEM $)$ as compared with those treated with saline $(14.1 \pm 0.8$, mean $\pm \operatorname{SEM})(F(1,45)=74.2$, $P<0.001)$.

\section{Discussion}

In Experiment 1, we demonstrated that extinction training procedures following heroin self-administration produced increased levels of DCX immunoreactivity in the DG, primarily in more rostral/dorsal regions, as compared to animals that underwent abstinence. Extinction of drugseeking behavior in rodent models of addiction is typically produced by withholding drug reinforcers and associated cues when animals are performing the same operant task that previously resulted in drug infusions and cue presentation. Although we did not assess the effects of extinction on the production of mature neurons in the DG, our findings are in line with those of Rapanelli and colleagues [35], who found that subjecting rats to an operant conditioning task resulted in increased numbers of mature neurons in the dorsal DG.

In Experiment 2, we found that impairment of cell proliferation in the DG by administration of GCV to GFAPtk mice resulted in increases in extinction responding during the first 3 sessions following cessation of heroin availability. Similarly, Noonan and colleagues [36] have previously demonstrated that ablation of both mature and immature neurons in the DG by hippocampal irradiation resulted in increased responding during the first 3 extinction sessions following cocaine self-administration. Unfortunately, however, the present study did not assess the impact of GCV treatment on formation of immature versus mature neurons, and only nonspecific quantification of the number of BrDU-positive cells as an indicator of cell proliferation was performed. Nonetheless the short time frame of GCV administration (10 days followed by commencement of extinction training on the following day) and the known 
amount of time required for the formation of mature neurons in the adult mouse DG ( $>4$ weeks, see $[37,38]$ ), our findings suggest that immature neurons in the DG may play a role in the process of extinction learning. Additional studies using transgenic lines of mice that more precisely allow the examination of the function on DG immature neurons, such as nestin-tk mice [24], are clearly warranted to confirm this.

We noted that extinction training following heroin selfadministration increased DCX immunoreactivity primarily in dorsal regions of the DG, while no such effects were observed in the ventral DG. Numerous lines of evidence suggest that dorsal regions of the hippocampus mediate drug-seeking behavior [39-41], although the precise role of adult-born neurons specifically in the DG, both immature and mature, has not yet been fully defined [17-19]. In contrast to our findings, other investigators have reported that cocaine self-administration followed by either continued drug intake or withdrawal results in preferential increases in DCX immunoreactivity in posterior/ventral regions of the DG [42]. The reasons underlying our observations of extinction-induced increases in DCX immunoreactivity in dorsal DG regions are currently unclear but may be related to the functional dichotomy of dorsal versus ventral areas of the hippocampus, with more dorsal regions mediating spatial information processing and more posterior/ventral regions mediating limbic functions such as emotionality [43]. Future studies employing methods to manipulate immature DG neurons with precise anatomical specificity are needed to resolve this issue.

There are several limitations of the present study. First, the number of DCX-positive neurons per coronal section in Experiment 1 is lower than those that have been reported by others $[36,42,44]$. There are numerous factors that could have contributed to these differences, such as opiate-induced suppression of NPC proliferation or survival [12, 45-47], different antigens to which DCX primary antisera were raised, and different methods of quantification (stereology versus manual counting). Second, due to technical problems with utilizing DCX antisera in mouse tissue, we were unable to quantify the degree to which GCV suppressed the formation of DCX-immunoreactive immature neurons in the DG, and only a nonspecific assessment of the number of proliferating cells by BrDU immunohistochemistry was performed. Clearly, future studies should be conducted to assess changes in a range of markers of NPC proliferation, differentiation, maturation, and cell death in the DG that occur as a result of extinction training. Third, both mice and rats self-administered heroin under limited access conditions ( $3 \mathrm{hr} /$ day) and were not likely opiate dependent, which is typically achieved under conditions of extended daily access $(6+\mathrm{hr} /$ day). The effects of extinction training on DCX immunoreactivity in the DG in heroin-dependent animals is an avenue worthy of further exploration.

In summary, we present evidence that extinction training following heroin self-administration increases the number of DCX-positive immature neurons selectively in the dorsal regions of the DG. We also present evidence that inhibition of cell proliferation in the DG increases responding during extinction, which is indicative of impaired extinction learning. These findings lend further support to the notion that immature neurons in the DG may play a functional role in certain learning and memory processes, including the extinction of drug-seeking behavior.

\section{Acknowledgment}

This work was supported by the Public Health Service Grant DA024355 from the National Institute on Drug Abuse.

\section{References}

[1] D. T. Balu and I. Lucki, "Adult hippocampal neurogenesis: regulation, functional implications, and contribution to disease pathology," Neuroscience and Biobehavioral Reviews, vol. 33, no. 3, pp. 232-252, 2009.

[2] G. L. Ming and H. Song, "Adult neurogenesis in the mammalian brain: significant answers and significant questions," Neuron, vol. 70, no. 4, pp. 687-702, 2011.

[3] M. Koehl and D. N. Abrous, "A new chapter in the field of memory: adult hippocampal neurogenesis," European Journal of Neuroscience, vol. 33, no. 6, pp. 1101-1114, 2011.

[4] W. Deng, J. B. Aimone, and F. H. Gage, "New neurons and new memories: how does adult hippocampal neurogenesis affect learning and memory?" Nature Reviews Neuroscience, vol. 11, no. 5, pp. 339-350, 2010.

[5] B. Leuner, E. Gould, and T. J. Shors, "Is there a link between adult neurogenesis and learning?" Hippocampus, vol. 16, no. 3, pp. 216-224, 2006.

[6] C. Zhao, W. Deng, and F. H. Gage, "Mechanisms and functional implications of adult neurogenesis," Cell, vol. 132, no. 4, pp. 645-660, 2008.

[7] M. F. Olive, Drug Addiction and Adult Neurogenesis, Research Signpost/Transworld Research Network Publishers, Karala, India, 2011.

[8] A. J. Eisch, H. A. Cameron, J. M. Encinas, L. A. Meltzer, G. L. Ming, and L. S. Overstreet-Wadiche, "Adult neurogenesis, mental health, and mental illness: hope or hype?" Journal of Neuroscience, vol. 28, no. 46, pp. 11785-11791, 2008.

[9] N. A. DeCarolis and A. J. Eisch, "Hippocampal neurogenesis as a target for the treatment of mental illness: a critical evaluation," Neuropharmacology, vol. 58, no. 6, pp. 884-893, 2010.

[10] G. Kempermann, J. Krebs, and K. Fabel, "The contribution of failing adult hippocampal neurogenesis to psychiatric disorders," Current Opinion in Psychiatry, vol. 21, no. 3, pp. 290-295, 2008.

[11] A. Kubesova, V. Bubenikova-Valesova, M. Mertlova, T. Palenicek, and J. Horacek, "Impact of psychotropic drugs on adult hippocampal neurogenesis," Neuroscience Research, vol. 73, no. 2, pp. 93-98, 2012.

[12] A. J. Eisch, M. Barrot, C. A. Schad, D. W. Self, and E. J. Nestler, "Opiates inhibit neurogenesis in the adult rat hippocampus," Proceedings of the National Academy of Sciences of the United States of America, vol. 97, no. 13, pp. 7579-7584, 2000.

[13] A. J. Eisch and G. C. Harburg, "Opiates, psychostimulants, and adult hippocampal neurogenesis: insights for addiction and stem cell biology," Hippocampus, vol. 16, no. 3, pp. 271-286, 2006. 
[14] K. Nixon, "Alcohol and adult neurogenesis: roles in Neurodegeneration and recovery in chronic alcoholism," Hippocampus, vol. 16, no. 3, pp. 287-295, 2006.

[15] T. A. Powrozek, Y. Sari, R. P. Singh, and F. C. Zhou, "Neurotransmitters and substances of abuse: effects on adult neurogenesis," Current Neurovascular Research, vol. 1, no. 3, pp. 251-260, 2004.

[16] A. Venkatesan, A. Nath, G. L. Ming, and H. Song, "Adult hippocampal neurogenesis: regulation by HIV and drugs of abuse," Cellular and Molecular Life Sciences, vol. 64, no. 16, pp. 2120-2132, 2007.

[17] J. J. Canales, "Adult neurogenesis and the memories of drug addiction," European Archives of Psychiatry and Clinical Neuroscience, vol. 257, no. 5, pp. 261-270, 2007.

[18] J. J. Canales, "Comparative neuroscience of stimulant-induced memory dysfunction: role for neurogenesis in the adult hippocampus," Behavioural Pharmacology, vol. 21, no. 5-6, pp. 379-393, 2010.

[19] C. D. Mandyam and G. F. Koob, "The addicted brain craves new neurons: putative role for adult-born progenitors in promoting recovery," Trends in Neurosciences, vol. 35, no. 4, pp. 250-260, 2012.

[20] C. Schmidt-Hieber, P. Jones, and J. Bischofberger, "Enhanced synaptic plasticity in newly generated granule cells of the adult hippocampus," Nature, vol. 429, no. 6988, pp. 184-187, 2004.

[21] S. Ge, C. H. Yang, K. S. Hsu, G. L. Ming, and H. Song, "A critical period for enhanced synaptic plasticity in newly generated neurons of the adult brain," Neuron, vol. 54, no. 4, pp. 559-566, 2007.

[22] J. P. Brown, S. Couillard-Després, C. M. Cooper-Kuhn, J. Winkler, L. Aigner, and H. G. Kuhn, "Transient expression of doublecortin during adult neurogenesis," Journal of Comparative Neurology, vol. 467, no. 1, pp. 1-10, 2003.

[23] O. Von Bohlen Und Halbach, "Immunohistological markers for staging neurogenesis in adult hippocampus," Cell and Tissue Research, vol. 329, no. 3, pp. 409-420, 2007.

[24] W. Deng, M. D. Saxe, I. S. Gallina, and F. H. Gage, "Adult-born hippocampal dentate granule cells undergoing maturation modulate learning and memory in the brain," Journal of Neuroscience, vol. 29, no. 43, pp. 13532-13542, 2009.

[25] K. M. Myers and W. A. Carlezon, "Extinction of drugand withdrawal-paired cues in animal models: relevance to the treatment of addiction," Neuroscience and Biobehavioral Reviews, vol. 35, no. 2, pp. 285-302, 2010.

[26] M. E. Bouton and D. Swartzentruber, "Sources of relapse after extinction in Pavlovian and instrumental learning," Clinical Psychology Review, vol. 11, no. 2, pp. 123-140, 1991.

[27] K. M. Kantak and B. A. Nic Dhonnchadha, "Pharmacological enhancement of drug cue extinction learning: translational challenges," Annals of the New York Academy of Sciences, vol. 1216, no. 1, pp. 122-137, 2011.

[28] M. E. Bouton, "A learning theory perspective on lapse, relapse, and the maintenance of behavior change," Health Psychology, vol. 19, no. 1, pp. 57-63, 2000.

[29] T. G. Bush, T. C. Savidge, T. C. Freeman et al., "Fulminant jejuno-ileitis following ablation of enteric gila in adult transgenic mice," Cell, vol. 93, no. 2, pp. 189-201, 1998.

[30] M. V. Sofroniew, T. G. Bush, N. Blumauer, Lawrence Kruger, L. Mucke, and M. H. Johnson, "Genetically-targeted and conditionally-regulated ablation of astroglial cells in the central, enteric and peripheral nervous systems in adult transgenic mice," Brain Research, vol. 835, no. 1, pp. 91-95, 1999.
[31] A. D. R. Garcia, N. B. Doan, T. Imura, T. G. Bush, and M. V. Sofroniew, "GFAP-expressing progenitors are the principal source of constitutive neurogenesis in adult mouse forebrain," Nature Neuroscience, vol. 7, no. 11, pp. 1233-1241, 2004.

[32] G. Paxinos and C. Watson, The Rat Brain in Stereotaxic Coordinates, Academic Press, San Diego, Calif, USA, 2007.

[33] J. M. Wojtowicz and N. Kee, "BrdU assay for neurogenesis in rodents," Nature Protocols, vol. 1, no. 3, pp. 1399-1405, 2006.

[34] A. J. Roberts, I. Y. Polis, and L. H. Gold, "Intravenous selfadministration of heroin, cocaine, and the combination in Balb/c mice," European Journal of Pharmacology, vol. 326, no. 2-3, pp. 119-125, 1997.

[35] M. Rapanelli, L. R. Frick, and B. S. Zanutto, "Learning an operant conditioning task differentially induces gliogenesis in the medial prefrontal cortex and neurogenesis in the hippocampus," PLoS ONE, vol. 6, no. 2, Article ID e14713, 2011.

[36] M. A. Noonan, S. E. Bulin, D. C. Fuller, and A. J. Eisch, "Reduction of adult hippocampal neurogenesis confers vulnerability in an animal model of cocaine addiction," Journal of Neuroscience, vol. 30, no. 1, pp. 304-315, 2010.

[37] M. S. Rao and A. K. Shetty, "Efficacy of doublecortin as a marker to analyse the absolute number and dendritic growth of newly generated neurons in the adult dentate gyrus," European Journal of Neuroscience, vol. 19, no. 2, pp. 234-246, 2004.

[38] J. S. Snyder, J. S. Choe, M. A. Clifford et al., "Adult-born hippocampal neurons are more numerous, faster maturing, and more involved in behavior in rats than in mice," Journal of Neuroscience, vol. 29, no. 46, pp. 14484-14495, 2009.

[39] R. A. Fuchs, K. A. Evans, C. C. Ledford et al., "The role of the dorsomedial prefrontal cortex, basolateral amygdala, and dorsal hippocampus in contextual reinstatement of cocaine seeking in rats," Neuropsychopharmacology, vol. 30, no. 2, pp. 296-309, 2005.

[40] R. A. Fuchs, J. L. Eaddy, Z. I. Su, and G. H. Bell, "Interactions of the basolateral amygdala with the dorsal hippocampus and dorsomedial prefrontal cortex regulate drug context-induced reinstatement of cocaine-seeking in rats," European Journal of Neuroscience, vol. 26, no. 2, pp. 487-498, 2007.

[41] D. R. Ramirez, G. H. Bell, H. C. Lasseter, X. Xie, S. A. Traina, and R. A. Fuchs, "Dorsal hippocampal regulation of memory reconsolidation processes that facilitate drug contextinduced cocaine-seeking behavior in rats," European Journal of Neuroscience, vol. 30, no. 5, pp. 901-912, 2009.

[42] M. A. Noonan, K. H. Choi, D. W. Self, and A. J. Eisch, "Withdrawal from cocaine self-administration normalizes deficits in proliferation and enhances maturity of adultgenerated hippocampal neurons," Journal of Neuroscience, vol. 28, no. 10, pp. 2516-2526, 2008.

[43] A. Sahay and R. Hen, "Adult hippocampal neurogenesis in depression," Nature Neuroscience, vol. 10, no. 9, pp. 11101115, 2007.

[44] A. Sankararaman, I. Masiulis, D. R. Richardson, J. M. Andersen, J. Mørland, and A. J. Eisch, "Methadone does not alter key parameters of adult hippocampal neurogenesis in the heroinnaïve rat," Neuroscience Letters, vol. 516, no. 1, pp. 99-104, 2012.

[45] L. Kahn, G. Alonso, E. Normand, and O. J. Manzoni, "Repeated morphine treatment alters polysialylated neural cell adhesion molecule, glutamate decarboxylase-67 expression and cell proliferation in the adult rat hippocampus," European Journal of Neuroscience, vol. 21, no. 2, pp. 493-500, 2005. 
[46] A. A. Arguello, S. J. Fischer, J. R. Schonborn, R. W. Markus, R. A. Brekken, and A. J. Eisch, "Effect of chronic morphine on the dentate gyrus neurogenic microenvironment," Neuroscience, vol. 159, no. 3, pp. 1003-1010, 2009.

[47] A. A. Arguello, G. C. Harburg, J. R. Schonborn, C. D. Mandyam, M. Yamaguchi, and A. J. Eisch, "Time course of morphine's effects on adult hippocampal subgranular zone reveals preferential inhibition of cells in S phase of the cell cycle and a subpopulation of immature neurons," Neuroscience, vol. 157, no. 1, pp. 70-79, 2008. 

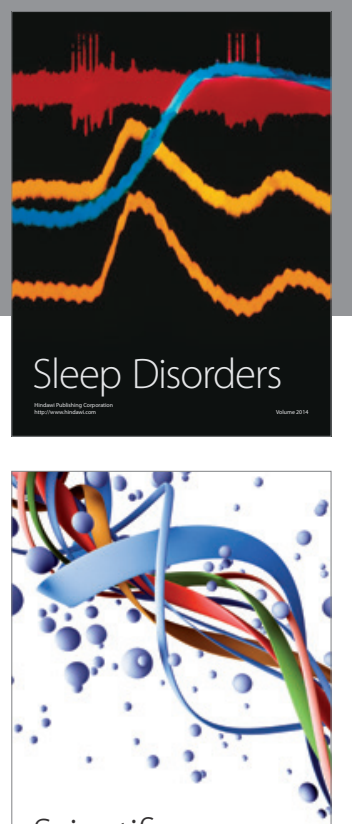

Scientifica
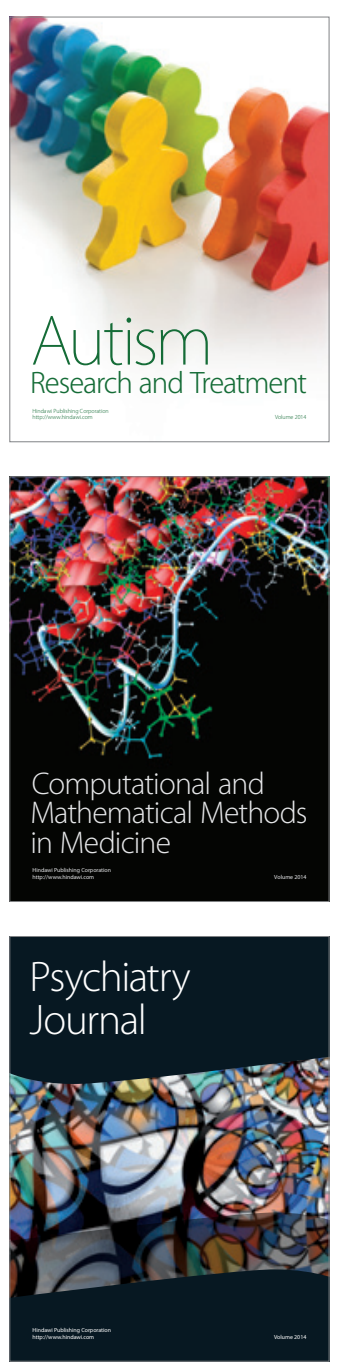
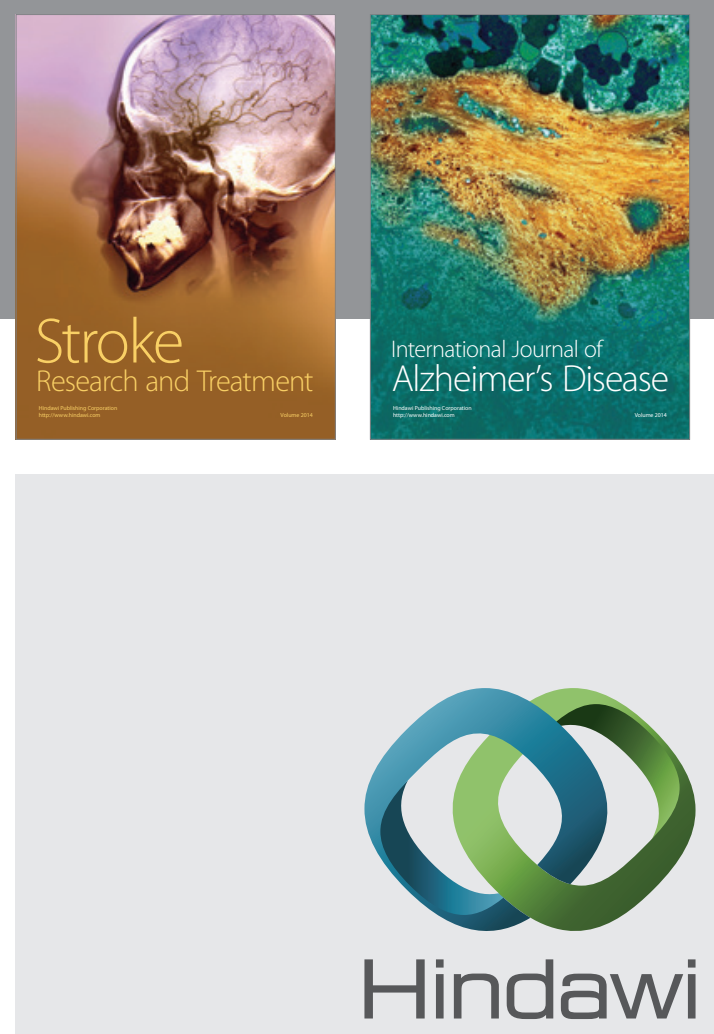

Submit your manuscripts at

http://www.hindawi.com
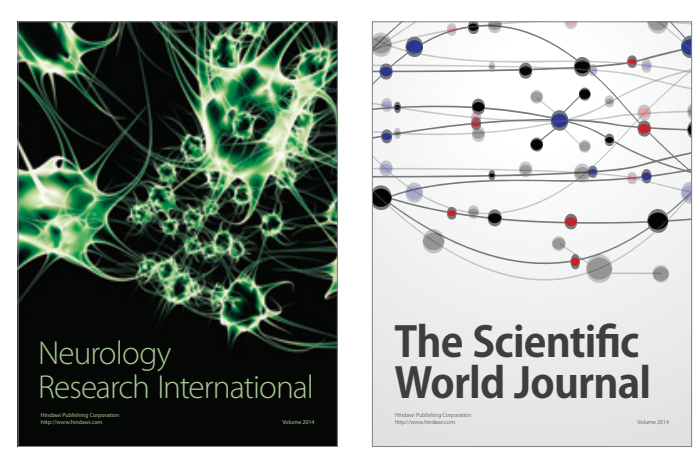

The Scientific World Journal

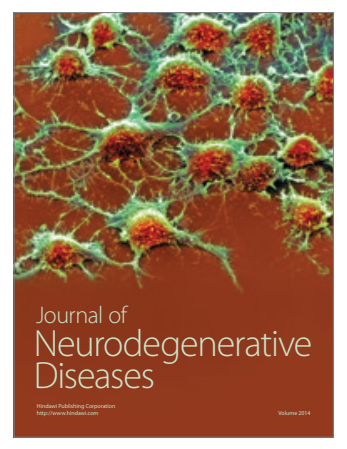

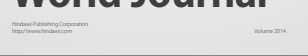

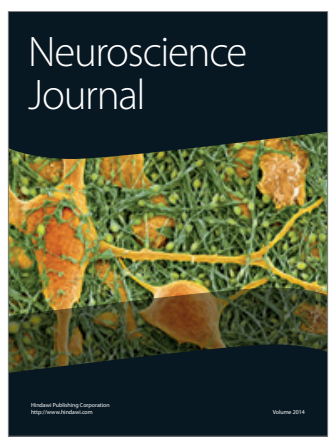

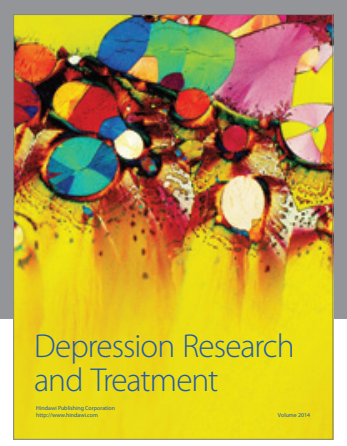
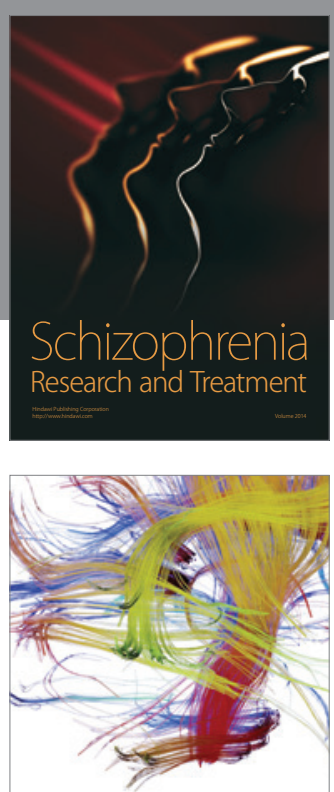

Brain Science

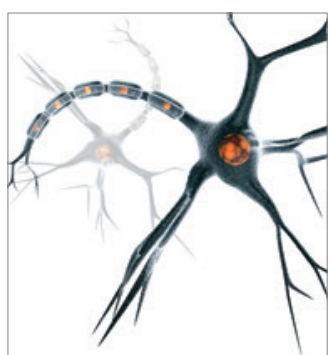

Neural Plasticity
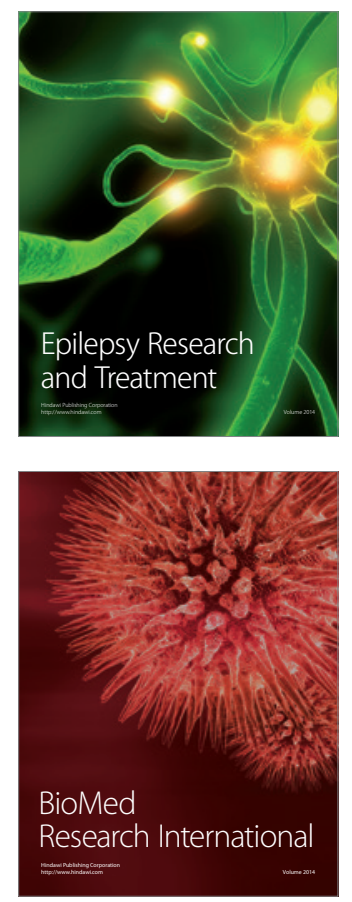

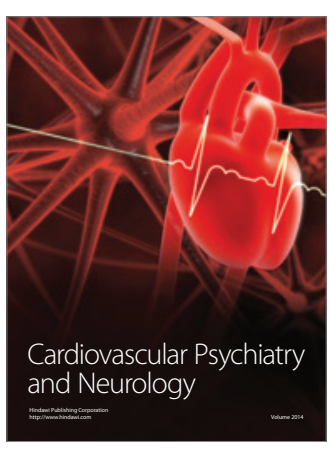

Parkinson's

Disease
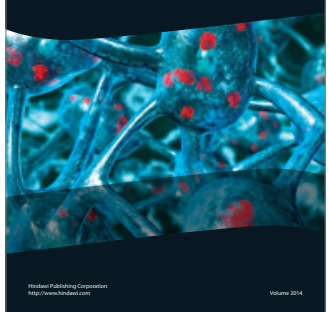